hep-th/0202025

$\mathrm{TIFR} / \mathrm{TH} / 02-03$

\title{
Non-Commutative Instantons and the Information Metric
}

\author{
Shahrokh Parvizi 用 \\ Department of Theoretical Physics, \\ Tata Institute of Fundamental Research, \\ Homi Bhabha Road, Mumbai 400 005, INDIA. \\ parvizi@theory.tifr.res.in
}

\begin{abstract}
By using the so-called Information Metric on the moduli space of an anti-selfdual (ASD) Instanton in a Self-Dual (SD) Non-Commutative background, we investigate the geometry of moduli space. The metric is evaluated perturbatively in non-commutativity parameter and we show that by putting a cut-off in the location of instanton in the definition of Information Metric we can recover the five dimensional space time in the presence of a B-field. This result shows that the non-commutative YM-Instanton Moduli corresponds to D-Instanton Moduli in the gravity side where the radial and transverse location of D-Instanton are corresponding to YM-Instanton size and location, respectively. The match is shown in the first order of non-commutativity parameter.
\end{abstract}

KEYWORDS: Instanton, Non-Commutative Gauge Theory, Moduli Space, AdS/CFT Correspondence.

PACS Numbers: 11.15.-q, 04.65.+e, 02.40.Gh

\footnotetext{
*On leave from "Institute for Studies in Theoretical Physics and Mathematics", Tehran, Iran.
} 


\section{Introduction}

In the study of AdS/CFT correspondence [1], the instantons are of particular interest as a non-perturbative probe. They entered in the subject through the work of Dorey, et $a l$ works [2], where they showed that, the Yang-Mills multi-instanton measure is related to the D-instanton counterpart in the gravity theory of the bulk. To reach this correspondence, the saddle point approximation is used for $S U(N)$ Yang-Mills in the large $N$ limit and the result is that the integral measure of YM instantons is equivalent to the $\mathrm{D}(-1)$-brane (D-Instanton) effective action in the bulk. Moreover in the large $N$ and large $k$ limit ( $k$ is the instantons number), the effective action corresponds to the $U(k)$ matrix model in IIB theory which, in turn is the D-Instanton effective action.

Since for $S U(N)$ gauge theory, every instanton lives in a $S U(2)$ subgroup, actually, the integration over zero modes of the gauge orientations in $S U(N)$ gives effectively the moduli of the instanton in $S U(2)$. Beside following the method of integration over zero modes and saddle point approximation for large $N$ limit, it is worth to ask for a direct relation between the moduli space of YM instantons on the boundary and the moduli space of D-instantons on the bulk.

However, we know that the moduli of the D-instanton is the $A d S_{5}$ itself, but it is not so clear for the YM instanton moduli. The most natural metric for the YM instanton moduli is the usual $L^{2}$ metric in the field configuration space. However, this metric for a single instanton on $\mathbf{R}^{4}$ is a flat metric and for an instanton on $S^{4}$ (which is the $A d S_{5}$ boundary) is a very complicated metric [3]. It means with $L^{2}$ metric the direct map between two moduli spaces in gauge theory and gravity sides is impossible. Therefore, we need a new definition for the metric on the moduli space.

Fortunately, as proposed by Blau, et al[ 4$]$, there exists an interesting metric on the YM instantons moduli called Information Metric (formerly introduced in [5]) which can do the job. This metric is constructed simply by the Instanton density $\operatorname{Tr}_{N} F^{2}$ (see (3.1)) and has the important property of conformal invariance which makes it a good candidate metric for the boundary theory.

It has been shown in [4], that the Information Metric for a single instanton on $\mathbf{R}^{4}$ (and also on $S^{4}$ ) is an $A d S_{5}$ metric in which the radial coordinate corresponds to the instanton size and the other transverse coordinates indicate the location of instanton in $\mathbf{R}^{\mathbf{4}}$. Therefore, this is the exact correspondence between YM instanton moduli space and the D-instanton location in the Supergravity Theory. Moreover, in [4], the authors consider possible small fluctuations of the base geometry around $\mathbf{R}^{\mathbf{4}}$ and find the correction to the $A d S_{5}$ metric. In this direction, one can think about another deformation of $\mathbf{R}^{\mathbf{4}}$, particularly non-commutative $\mathbf{R}_{\mathbf{N C}}^{4}$, and look for the corresponding instanton moduli and compare it with the Supergravity side.

On the gravity side, it is worth mentioning the work of Maldacena and Russo [6] in which the AdS/CFT correspondence is understood as correspondence of the noncommutative YM theories in the boundary to Supergravity with anti-symmetric $B$ field in the bulk. The latter has an Einstein metric (in selfdual $B$ background [7]) and contains the D-instantons. The non-commutativity is related to the boundary value of $1 / B$. So in this context, it is quiet natural to expect that an extension of YM instantons to the $\mathbf{R}_{\mathbf{N C}}^{4}$ background may have a correspondence to the Supergravity D-instantons in the 
Maldacena-Russo background.

On the other hand, in the gauge theory side, the non-commutative instantons are also well-known. The general prescription, as a non-commutative version of ADHM construction [8], introduced in the work by Nekrasov and Schwarz in [9] and the more explicit solutions for the non-commutative ADHM equations are discussed in several works 110, 11, 12, 13, 14, 15, 16, 17, 18].

The purpose of this letter is to extend the idea of the Information Metric to the NC single $S U(N)$ instanton and find the relation with the D-instanton in [6], if any.

The extension needs a careful definition for the Information Metric in the NC background. To overcome some technical problems and to recover the commutative case, we study the Information Metric in the small non-commutativity limit. By proposing a modified definition for the Information Metric, we can relate it to the Supergravity metric in the first order of approximation.

\section{Non-Commutative Instantons}

In the following subsections, the instanton construction in a non-commutative space is reviewed along which we introduce our notations.

\subsection{Non-Commutative $\mathbf{R}^{4}$}

Let us start by the non-commutative Euclidean four dimensional space, $\mathbf{R}_{\mathbf{N C}}^{4}$. In general, the non-commutativity is defined by the commutator of coordinates as follows,

$$
\left[x^{\mu}, x^{\nu}\right]=i \theta^{\mu \nu}
$$

where $\theta$ is a real anti-symmetric constant tensor. If we consider $\theta$ to be a rank 4 tensor, then by four dimensional rotations and re-scaling, it is possible to transform it to the following form,

$$
\theta^{12}= \pm \theta^{34}
$$

with the other components equal to zero. In (2.2), the + and - signs define self-dual and anti-selfdual $\theta$ tensor, respectively. We use self-dual $\theta$ in this letter and set $\theta^{12}=\theta^{34} \equiv$ $\zeta / 2$. I

It is also convenient to use complex coordinates defined as:

$$
\begin{aligned}
& z_{1}=x_{2}+i x_{1}, \quad \bar{z}_{1}=x_{2}-i x_{1}, \\
& z_{2}=x_{4}+i x_{3}, \quad \bar{z}_{2}=x_{4}-i x_{3} .
\end{aligned}
$$

Then, the commutation relations will be

$$
\left[z_{i}, \bar{z}_{j}\right]=-\zeta \delta_{i j}
$$

\footnotetext{
${ }^{\dagger}$ It can be converted to the anti-self-dual case by a parity transformation.
} 
The above commutators have a harmonic oscillator representation in which coordinates $z$ act as operators on the basis $\left|n_{1}, n_{2}\right\rangle$ as follows,

$$
\begin{aligned}
z_{1}\left|n_{1}, n_{2}\right\rangle & =\sqrt{\zeta} \sqrt{n_{1}+1}\left|n_{1}+1, n_{2}\right\rangle, \quad \bar{z}_{1}\left|n_{1}, n_{2}\right\rangle=\sqrt{\zeta} \sqrt{n_{1}}\left|n_{1}-1, n_{2}\right\rangle, \\
z_{2}\left|n_{1}, n_{2}\right\rangle & =\sqrt{\zeta} \sqrt{n_{2}+1}\left|n_{1}, n_{2}+1\right\rangle, \quad \bar{z}_{2}\left|n_{1}, n_{2}\right\rangle=\sqrt{\zeta} \sqrt{n_{2}}\left|n_{1}, n_{2}-1\right\rangle,
\end{aligned}
$$

and as usual:

$$
z_{i} \bar{z}_{i}\left|n_{1}, n_{2}\right\rangle=\zeta n_{i}\left|n_{1}, n_{2}\right\rangle, \quad(i=1,2 \text { no } \text { sum }) .
$$

The relation between operator valued functions and ordinary c-functions can be obtained by the following map:

$$
\Omega: \hat{\phi}(\hat{z}, \hat{\bar{z}}) \rightarrow \tilde{\phi}(z, \bar{z})=\langle z|\hat{\phi}(\hat{z}, \hat{\bar{z}})| \bar{z}\rangle,
$$

where $|\bar{z}\rangle$ and $\langle z|$ are the normalized coherent states,

$$
\hat{\bar{z}}|\bar{z}\rangle=\bar{z}|\bar{z}\rangle, \quad\langle z| \hat{z}=\langle z| z, \quad\langle z \mid \bar{z}\rangle=1
$$

In this map, one can also relate sum over states to an integral over functions as follows,

$$
\Omega:(\zeta \pi)^{2} \operatorname{Tr}_{\mathcal{H}} \hat{\phi}(\hat{z}, \hat{\bar{z}}) \rightarrow \int d^{4} z \tilde{\phi}(z, \bar{z})
$$

where $\mathcal{H}$ is the representing Hilbert space.

\subsection{The ADHM Construction of Instantons}

SD or ASD instantons are defined as field configurations for SD or ASD Yang-Mills field, respectively, and subject to the constraint,

$$
Q:=-\frac{1}{16 \pi^{2}} \int d^{4} x \operatorname{Tr}_{N} F \wedge F= \pm k
$$

where $Q$ is known as the instanton charge and its absolute value is an integer constant $k$. The sign \pm corresponds to SD or ASD instantons, respectively.

The solutions can be found by an algebraic ansatz so-called ADHM construction [8]. Here, we briefly review the construction.

Firstly, consider an $(N+2 k) \times(2 k)$ matrix $\Delta_{[N+2 k] \times[2 k]}$ which is linear in coordinates as follows,

$$
\begin{aligned}
& \Delta_{[N+2 k] \times[2 k]}=a_{[N+2 k] \times[2 k]}+b_{[N+2 k] \times[2 k]} x_{[2] \times[2]} \quad \text { for SD Instantons } \\
& \Delta_{[N+2 k] \times[2 k]}=a_{[N+2 k] \times[2 k]}+b_{[N+2 k] \times[2 k]} \bar{x}_{[2] \times[2]} \quad \text { for ASD Instantons }
\end{aligned}
$$

where $a$ and $b$ are constant complex valued matrices and we have used quaternionic representation for coordinates $x_{\mu}$,

$$
\bar{x}=\left(\begin{array}{cc}
-\bar{z}_{1} & z_{2} \\
-\bar{z}_{2} & -z_{1}
\end{array}\right), \quad x=\left(\begin{array}{cc}
-z_{1} & -z_{2} \\
\bar{z}_{2} & -\bar{z}_{1}
\end{array}\right)
$$


Since $\Delta_{[N+2 k] \times[2 k]}$ has $N$ more rows than columns, it has an $N$ dimensional null space. We put independent null vectors of $\bar{\Delta}$ in an $(N+2 k) \times N$ dimensional matrix $U(x)$, i.e.,

$$
\begin{aligned}
& \bar{\Delta}_{[2 k] \times[N+2 k]} U_{[N+2 k] \times[N]}=0 \\
& \bar{U}_{[N] \times[N+2 k]} \Delta_{[N+2 k] \times[2 k]}=0
\end{aligned}
$$

with the normalization condition,

$$
\bar{U}_{[N] \times[N+2 k]} U_{[N+2 k] \times[N]}=1_{[N] \times[N]} .
$$

Then the gauge potential in this ansatz will be derived from $U$ as follows,

$$
A_{\mu[N] \times[N]}=\bar{U}_{[N] \times[N+2 k]} \partial_{\mu} U_{[N+2 k] \times[N]}
$$

To have an ASD or SD $F_{\mu \nu}$ for non-zero $k$, we need two additional conditions.

$$
\begin{gathered}
\bar{\Delta}_{[2 k] \times[N+2 k]} \Delta_{[N+2 k] \times[2 k]}=1_{[2] \times[2]]} f_{[k] \times[k]}^{-1} \\
\Delta_{[N+2 k] \times[2 k]} f_{[k] \times[k]} \bar{\Delta}_{[2 k] \times[N+2 k]}+U_{[N+2 k] \times[N]} \bar{U}_{[N] \times[N+2 k]}=1_{[N+2 k] \times[N+2 k]}
\end{gathered}
$$

where $f$ is an arbitrary $x$-dependent $k \times k$ matrix. Using the above relations, it is easy to write the instanton field strength as,

$$
\begin{aligned}
F_{\mu \nu}^{A S D} & =4 \bar{U} b \bar{\sigma}_{\mu \nu} f \bar{b} U \\
F_{\mu \nu}^{S D} & =4 \bar{U} b \sigma_{\mu \nu} f \bar{b} U
\end{aligned}
$$

where

$$
\bar{\sigma}_{\mu \nu}=\frac{1}{2} i \bar{\eta}_{\mu \nu}^{a} \tau^{a} \quad \text { and } \quad \sigma_{\mu \nu}=\frac{1}{2} i \eta_{\mu \nu}^{a} \tau^{a}
$$

with $\eta_{\mu \nu}^{a}$ 's the standard 'tHooft symbols and $\tau^{a}$ the Pauli matrices. The anti-selfduality (selfduality) of $F_{\mu \nu}$ comes simply from anti-selfduality (selfduality) of $\bar{\sigma}_{\mu \nu}\left(\sigma_{\mu \nu}\right)$, respectively.

The above construction is valid for non-commutative case too. In the non-commutative case we must care about the coordinates which are operators. To be more specific, we convert $\bar{\Delta}$ to the following matrix form,

$$
\bar{\Delta} \equiv\left(\begin{array}{ccc}
I & B_{1}^{\dagger}-\bar{z}_{1} & -B_{2}+z_{2} \\
J^{\dagger} & B_{2}^{\dagger}-\bar{z}_{2} & B_{1}-z_{1}
\end{array}\right)
$$

where $I_{[k] \times[N+k]}, J_{[N+k] \times[k]}$ and $B_{[k] \times[k]}$ are constant matrices including in the matrix $a_{[N+2 k] \times[2 k]}$ in (2.13). Then the condition (2.19) is equivalent to the following equations known as ADHM equations in the non-commutative space [9],

$$
\begin{gathered}
{\left[B_{1}, B_{1}^{\dagger}\right]+\left[B_{2}, B_{2}^{\dagger}\right]+I I^{\dagger}-J^{\dagger} J=\left[z_{1}, \bar{z}_{1}\right]+\left[z_{2}, \bar{z}_{2}\right]=-\zeta} \\
{\left[B_{1}, B_{2}^{\dagger}\right]+I J^{\dagger}=0}
\end{gathered}
$$




\section{$2.3 k=1$ Instantons}

The main subject in the ADHM ansatz is to construct $\Delta$ and $U$ such that the conditions $(2.19)$ and (2.20) are satisfied. Even in the commutative case, it is difficult to solve the problem for an arbitrary $k$. Here we recall the final solution for one instanton $(k=1)$ in the commutative $\mathbf{R}^{\mathbf{4}}$ :

$$
\begin{aligned}
& F^{\mu \nu}=-4 \eta_{\mu \nu}^{a} \frac{\rho^{2}}{\left[(x-a)^{2}+\rho^{2}\right]^{2}} \\
& \operatorname{Tr}_{N} F^{2}=96 \frac{\rho^{4}}{\left[(x-a)^{2}+\rho^{2}\right]^{4}}
\end{aligned}
$$

where $\rho$ and $a^{\mu}$ are the instanton size and location, respectively. These parameters are usually encoded in the matrix $a_{[N+2 k] \times[2 k]}$.

For the non-commutative $\mathbf{R}_{\mathbf{N C}}^{4}$ and $k=1$, we look at the ASD instanton in the SD non-commutative backgroundf and according to [14] (see also [15]) the result will be as follows,

$$
\operatorname{Tr}_{N} F^{2}=S_{1} p+S_{2}
$$

where the instanton is located at the origin and

$$
\begin{aligned}
& S_{1}:=4\left(\left(C_{1}^{2}+C_{4}^{2}\right) z^{2}\left(z^{2}+\zeta\right)+2 C_{2}^{2}\left(z^{2}-\frac{\zeta}{2}\right) z^{2}\right) \\
& S_{2}:=8 C_{3}^{2}\left(z^{2}+\zeta\right)\left(z^{2}+\frac{3 \zeta}{2}\right) .
\end{aligned}
$$

with

$$
\begin{aligned}
C_{1} & :=\frac{-2\left(\rho^{2}+\zeta\right)}{z^{2}\left(z^{2}+\rho^{2}+\frac{\zeta}{2}\right)\left(z^{2}+\rho^{2}+\zeta\right)} \\
C_{2} & :=\frac{2 \rho \sqrt{\rho^{2}+\zeta}}{z^{2}\left(z^{2}+\rho^{2}+\frac{\zeta}{2}\right)\left(z^{2}+\rho^{2}+\zeta\right)}\left(\frac{z^{2}+\rho^{2}+\zeta}{z^{2}+\rho^{2}}\right)^{\frac{1}{2}} \\
C_{3} & :=\frac{2 \rho \sqrt{\rho^{2}+\zeta}}{\left(z^{2}+\zeta\right)\left(z^{2}+\rho^{2}+\frac{3 \zeta}{2}\right)\left(z^{2}+\rho^{2}+\zeta\right)}\left(\frac{z^{2}+\rho^{2}+\zeta}{z^{2}+\rho^{2}+2 \zeta}\right)^{\frac{1}{2}} \\
C_{4} & :=\frac{-2 \rho^{2}}{\left(z^{2}+\zeta\right)\left(z^{2}+\rho^{2}+\frac{3 \zeta}{2}\right)\left(z^{2}+\rho^{2}+\zeta\right)} \\
z^{2} & :=z_{1} \bar{z}_{1}+z_{2} \bar{z}_{2}
\end{aligned}
$$

and $p$ is a projection operator which excludes $|0,0\rangle$ state. The matrix representation of $F^{2}$ in $\left|n_{1}, n_{2}\right\rangle$ basis is obtained simply by replacing $z^{2}$ with $\zeta n / 2 \equiv \zeta / 2\left(n_{1}+n_{2}\right)$ and it

†The moduli of ASD instantons on ASD background is equivalent to the commutative case, since the instanton equations in both commutative cases and non-commutative cases are the same by the Seiberg-Witten map [19]. 
will be diagonal as expected from the radial symmetry of $F^{2}$ in four dimensional space $\mathbf{R}^{4}$.

It can be shown that the instanton charge for this case is indeed exactly -1 , [14, 15],

$$
\begin{aligned}
Q=\frac{-1}{16 \pi^{2}} \int d^{4} z \operatorname{Tr}_{N} F^{2}\left(z^{2}\right) & =\frac{-1}{16 \pi^{2}}\left(\frac{\zeta \pi}{2}\right)^{2} \sum_{n_{1}, n_{2}}\left\langle n_{1}, n_{2}\left|\operatorname{Tr}_{N} \hat{F}^{2}\right| n_{1}, n_{2}\right\rangle \\
& =\frac{-\zeta^{2}}{64} \sum_{n}(n+1) F^{2}(\zeta n / 2)=-1
\end{aligned}
$$

where $F^{2}(\zeta n / 2)$ is the matrix element of $\operatorname{Tr}_{N} \hat{F}^{2}$.

\section{The Information Metric}

As stated in the introduction, the Information Metric is an alternative to construct the geometry of the instantons moduli space. It can be defined in terms of the field density as follows,

$$
\begin{aligned}
G_{A B} & :=\frac{5}{256 \pi^{2}} \int d^{4} z F^{2} \partial_{A} \log F^{2} \partial_{B} \log F^{2} \\
& =\frac{5}{256 \pi^{2}} \int d^{4} z \frac{\partial_{A} F^{2} \partial_{B} F^{2}}{F^{2}}
\end{aligned}
$$

where $F^{2} \equiv \operatorname{Tr}_{N} F^{2}$ and $\partial_{A} \equiv \partial / \partial y^{A}$ in which $y^{A}$ are the moduli space parameters and correspond to the location and size of instantons, $y^{A}=\left(a^{\mu}, \rho\right)$.

Firstly, let us quote from [四], the resulting metric for the commutative single instanton (2.27),

$$
d s^{2}=\frac{1}{\rho^{2}}\left(d \vec{a}^{2}+d \rho^{2}\right)
$$

The above metric is the well-known Euclidean $\mathrm{AdS}_{5}$ metric. This result shows that the moduli space of YM-Instanton equipped with the Information Metric has exactly the bulk geometry in the AdS/CFT context. This is the most direct correspondence between YM-Instantons and D-Instantons. In the following, we consider possible definitions of the Information Metric for the NC YM-Instantons to explore this correspondence more.

The natural extension of the Information Metric to a non-commutative instanton, is to replace the commutative $F^{2}$ in (3.1) by its operator version in non-commutative case (2.29). The integration is also replaced by a Trace over Hilbert Space of non-commutative space. So, we start with the following definition:

$$
G_{A B}:=\frac{5}{256 \pi^{2}}\left(\frac{\zeta \pi}{2}\right)^{2} \operatorname{Tr}_{\mathcal{H}} \hat{F}^{2} \partial_{A} \log \hat{F}^{2} \partial_{B} \log \hat{F}^{2}
$$

or

$$
G_{A B}:=\frac{5}{256 \pi^{2}}\left(\frac{\zeta \pi}{2}\right)^{2} \operatorname{Tr}_{\mathcal{H}} \frac{1}{\hat{F}^{2}} \partial_{A} \hat{F}^{2} \partial_{B} \hat{F}^{2} .
$$


The first difficulty arises here with the ambiguity in ordering of the terms. We can use the symmetry between the indices $A$ and $B$ in the metric to fix the position of $\partial_{A} F^{2}$ and $\partial_{B} F^{2}$ by symmetrizing them. The position of $\frac{1}{F^{2}}$ is still ambiguous, and we can consider either of two cases bellow,

$$
\begin{aligned}
G_{A B}^{(1)} & :=\frac{5}{256 \pi^{2}}\left(\frac{\zeta \pi}{2}\right)^{2} \operatorname{Tr}_{\mathcal{H}} \frac{1}{\hat{F}^{2}} \partial_{\{A} \hat{F}^{2} \partial_{B\}} \hat{F}^{2} \\
G_{A B}^{(2)} & :=\frac{5}{256 \pi^{2}}\left(\frac{\zeta \pi}{2}\right)^{2} \operatorname{Tr}_{\mathcal{H}} \partial_{\{A} \hat{F}^{2} \frac{1}{\hat{F}^{2}} \partial_{B\}} \hat{F}^{2} .
\end{aligned}
$$

However, it can be shown that for the radially symmetric class of solutions (including $(2.29)$ ), both $G_{A B}^{(1)}$ and $G_{A B}^{(2)}$ have the same result. Moreover, the Information Metric is diagonal in this case.

To calculate the Information Metric, firstly, we need the derivatives of $F^{2}$. Here, we put the instanton at the point with complex coordinates $\left(a_{i}, \bar{a}_{i}\right)$, now taking

$$
\begin{aligned}
& z_{i}=x_{i}-a_{i} \\
& \bar{z}_{i}=\bar{x}_{i}-\bar{a}_{i} .
\end{aligned}
$$

Note that the non-commutative algebra (2.5) is valid for both $z$ and $x$ coordinates. Then we have the following derivatives involved in the Information Metric:

$$
\begin{aligned}
& \frac{\partial \hat{F}^{2}}{\partial a_{i}}=-\frac{\partial \hat{F}^{2}}{\partial z_{i}}=-\frac{2}{\zeta}\left[\bar{z}_{i}, \hat{F}^{2}\right] \\
& \frac{\partial \hat{F}^{2}}{\partial \bar{a}_{i}}=-\frac{\partial \hat{F}^{2}}{\partial \bar{z}_{i}}=\frac{2}{\zeta}\left[z_{i}, \hat{F}^{2}\right] \\
& \frac{\partial \hat{F}^{2}}{\partial \rho}
\end{aligned}
$$

We use the harmonic oscillator basis to find the matrix elements of terms in (3.5) as follows,

$$
\begin{aligned}
\left\langle m_{1}, m_{2}\left|\frac{1}{\hat{F}^{2}}\right| n_{1}, n_{2}\right\rangle & =\frac{1}{F^{2}(\zeta n / 2)} \delta_{\vec{m} \vec{n}} \\
\left\langle m_{1}, m_{2}\left|\frac{\partial \hat{F}^{2}}{\partial \rho}\right| n_{1}, n_{2}\right\rangle & =\frac{\partial F^{2}(\zeta n / 2)}{\partial \rho} \delta_{\vec{m} \vec{n}} \\
\left\langle m_{1}, m_{2}\left|\frac{\partial \hat{F}^{2}}{\partial a_{1}}\right| n_{1}, n_{2}\right\rangle & =\sqrt{\frac{2}{\zeta}}\left(W(n-1) \sqrt{n_{1}} \delta_{m_{1}+1, n_{1}} \delta_{m_{2} n_{2}}-S_{1}(0) \delta_{\vec{m} 0} \delta_{n_{1} 1} \delta_{n_{2} 0}\right) \\
\left\langle m_{1}, m_{2}\left|\frac{\partial \hat{F}^{2}}{\partial a_{2}}\right| n_{1}, n_{2}\right\rangle & =\sqrt{\frac{2}{\zeta}}\left(W(n-1) \sqrt{n_{2}} \delta_{m_{2}+1, n_{2}} \delta_{m_{1} n_{1}}-S_{1}(0) \delta_{\vec{m} 0} \delta_{n_{2} 1} \delta_{n_{1} 0}\right) \\
\left\langle m_{1}, m_{2}\left|\frac{\partial \hat{F}^{2}}{\partial \bar{a}_{1}}\right| n_{1}, n_{2}\right\rangle & =\sqrt{\frac{2}{\zeta}}\left(W(n) \sqrt{n_{1}+1} \delta_{m_{1}-1, n_{1}} \delta_{m_{2} n_{2}}-S_{1}(0) \delta_{\vec{m} 0} \delta_{n_{1} 1} \delta_{n_{2} 0}\right) \\
\left\langle m_{1}, m_{2}\left|\frac{\partial \hat{F}^{2}}{\partial \bar{a}_{2}}\right| n_{1}, n_{2}\right\rangle & =\sqrt{\frac{2}{\zeta}}\left(W(n) \sqrt{n_{2}+1} \delta_{m_{2}-1, n_{2}} \delta_{m_{1} n_{1}}-S_{1}(0) \delta_{\vec{m} 0} \delta_{n_{1} 0} \delta_{n_{2} 1}\right)
\end{aligned}
$$


where $W(n) \equiv S_{1}(\zeta n / 2)+S_{2}(\zeta n / 2)$ and $n=n_{1}+n_{2}$.

Now, one can write the Information Metric as follows, 8

$$
G_{A B}=\frac{5}{256 \pi^{2}}\left(\frac{\zeta \pi}{2}\right)^{2} \sum_{\vec{n}, \vec{m}, \vec{k}}^{\infty}\left\langle\vec{n}\left|\frac{1}{F^{2}}\right| \vec{m}\right\rangle\left\langle\vec{m}\left|\partial_{\{A} F^{2}\right| \vec{k}\right\rangle\left\langle\vec{k}\left|\partial_{B\}} F^{2}\right| \vec{n}\right\rangle
$$

The sums over two indices, say $m$ and $k$, can be done easily by the help of Kronecker delta functions in (3.9) and the non-vanishing components will be as follows,

$$
\begin{aligned}
& G_{\rho \rho}=\frac{5}{256} \frac{\zeta^{2}}{4}\left(\sum_{n_{1}, n_{2} \neq(0,0)}^{\infty} \frac{(\partial W(n) / \partial \rho)^{2}}{W(n)}+H_{0}\right) \\
&=\frac{5}{256} \frac{\zeta^{2}}{4}\left(\sum_{n=1}^{\infty}(n+1) \frac{(\partial W(n) / \partial \rho)^{2}}{W(n)}+H_{0}\right) \\
& G_{a_{i} \bar{a}_{i}}=\frac{5}{256} \frac{\zeta^{2}}{4}\left(\sum_{n_{1}, n_{2} \neq(0,0)}^{\infty} \frac{2}{\zeta} \frac{n_{i}(T(n-1))^{2}+\left(n_{i}+1\right)(T(n))^{2}}{2 W(n)}+G_{0}\right) \\
&=\frac{5}{256} \frac{\zeta^{2}}{4}\left(\sum_{n=1}^{\infty}(n+1) \frac{2}{\zeta} \frac{n(T(n-1))^{2}+(n+2)(T(n))^{2}}{4 W(n)}+G_{0}\right)
\end{aligned}
$$

where,

$$
\begin{aligned}
& H_{0}=\frac{\left(\partial S_{2}(0) / \partial \rho\right)^{2}}{S_{2}(0)} \\
& G_{0}=\frac{2}{\zeta}\left(\frac{\left(S_{1}(0)\right)^{2}-2 T(0) S_{1}(0)}{2 W(1)}+\frac{\left(T(0)-S_{1}(0)\right)^{2}}{2 S_{2}(0)}\right)
\end{aligned}
$$

and $T(n)=W(n)-W(n+1)$. We have also used the following identities for summations over $n_{1}$ and $n_{2}$,

$$
\sum_{n_{1}, n_{2}}^{\infty} g(n) n_{1}=\sum_{n_{1}, n_{2}}^{\infty} g(n) n_{2}=\sum_{n_{1}, n_{2}}^{\infty} g(n) n / 2=\sum_{n}^{\infty}(n+1) g(n) n / 2
$$

In contrast to (2.31), the resulting sum over $n$ for the Information Metric is too complicated to have an interesting interpretation. Here, we consider small non-commutativity approximation. Firstly, for the limit $\zeta \rightarrow 0$, the sums over $n$ go to an integral. It can be seen for a continuous function $g$ as follows,

$$
\lim _{\zeta \rightarrow 0} \sum_{n=1}^{\infty}(n+1) g(n \zeta / 2)=\frac{2}{\zeta} \int_{\zeta / 2}^{\infty} d t\left(\frac{2 t}{\zeta}+1\right) g(t)+\mathcal{O}\left(\zeta^{2}\right)
$$

This approximation is valid up to the first order in $\zeta$. To check this limit, we apply it to the instanton charge formula (2.31),

$$
\begin{aligned}
Q & =\frac{-\zeta^{2}}{64} \sum_{n}(n+1) F^{2}(\zeta n / 2)=\frac{-\zeta^{2}}{64} \frac{2}{\zeta} \int_{\zeta / 2}^{\infty} d t\left(\frac{2 t}{\zeta}+1\right) F^{2}(t)+\mathcal{O}\left(\zeta^{2}\right) \\
& =-1+\mathcal{O}\left(\zeta^{2}\right)
\end{aligned}
$$

\footnotetext{
${ }^{\S}$ As mentioned before, we know that in the end of calculation we will have $G_{A B}^{(1)}=G_{A B}^{(2)} \equiv G_{A B}$.
} 
After converting the series in (3.11) and (3.12) to integrals, the components of the Information Metric can be obtained by expanding the integrands in a power series of $\zeta$ (while fixing $t$ ). The results are in the following,

$$
\begin{aligned}
G_{\rho \rho} & =\frac{5}{256} \frac{\zeta^{2}}{4}\left(\frac{2}{\zeta} \int_{\zeta / 2}^{\infty}\left(\frac{1536\left(\rho^{3}-\rho t\right)^{2}}{\left(\rho^{2}+t\right)^{6}}-\frac{9216\left(\rho^{3}-\rho t\right)^{2} \zeta}{\left(\rho^{2}+t\right)^{7}}+\mathcal{O}\left(\zeta^{2}\right)\right)\left(\frac{2 t}{\zeta}+1\right) d t+H_{0}\right) \\
& =\frac{1}{\rho^{2}}\left(1-\frac{\zeta}{\rho^{2}}+\mathcal{O}\left(\frac{\zeta^{2}}{\rho^{4}}\right)\right) \\
G_{a_{i} \bar{a}_{i}} & =\frac{5}{256} \frac{\zeta^{2}}{4}\left(\frac{2}{\zeta} \int_{\zeta / 2}^{\infty}\left(\frac{192 t \rho^{4}}{\left(\rho^{2}+t\right)^{6}}+\frac{96\left(2 t^{2} \rho^{2}-9 t \rho^{4}+\rho^{6}\right) \zeta}{\left(\rho^{2}+t\right)^{7}}+\mathcal{O}\left(\zeta^{2}\right)\right)\left(\frac{2 t}{\zeta}+1\right) d t+G_{0}\right) \\
& =\frac{1}{2 \rho^{2}}\left(1-\frac{\zeta}{2 \rho^{2}}+\frac{45 \zeta}{64 \rho^{2}}+\mathcal{O}\left(\frac{\zeta^{2}}{\rho^{4}}\right)\right)
\end{aligned}
$$

where the third term in the last equation is the contribution of $G_{0}$. Finally, we have the following metric,

$$
d s^{2}=\left(1+\frac{13 \zeta}{64 \rho^{2}}+\mathcal{O}\left(\frac{\zeta^{2}}{\rho^{4}}\right)\right) \frac{d \vec{a}^{2}}{\rho^{2}}+\left(1-\frac{\zeta}{\rho^{2}}+\mathcal{O}\left(\frac{\zeta^{2}}{\rho^{4}}\right)\right) \frac{d \rho^{2}}{\rho^{2}}
$$

Unfortunately, this metric does not agree with the expected results in Supergravity side. Actually, if we neglect the contribution of $G_{0}$, it will be in agreement with the SUGRA result (see section 4). This means excluding the ground state $|0,0\rangle$ from $\operatorname{Tr}_{\mathcal{H}}$ in the definitions of Information Metric. In the coherent states language, the $\operatorname{Tr}_{\mathcal{H}}$ is replaced by integral over $z$ coordinates and the exclusion of $|0,0\rangle$ state is equivalent to excluding the origin from the integration region. So we can use the following expression as Modified Information Metric,

$$
\begin{aligned}
\tilde{G}_{A B} & =\frac{5}{256 \pi^{2}} \int_{\mathcal{M}^{\prime}} d^{4} z\langle z| \frac{1}{\hat{F}^{2}} \partial_{\left\{{ }_{A} \hat{F}^{2} \partial_{B\}} \hat{F}^{2}|\bar{z}\rangle\right.} \\
& =\frac{5}{256 \pi^{2}} \int_{\mathcal{M}^{\prime}} d^{4} z \widetilde{\left(\frac{1}{F^{2}}\right)} * \widetilde{\partial_{\left\{A_{F} F^{2}\right.} * \widetilde{\partial_{B\}} F^{2}}}
\end{aligned}
$$

where $\mathcal{M}^{\prime}=\mathbf{R}^{\mathbf{4}}-\mathbf{B}^{\mathbf{0}}(\eta \zeta / 2)$ and $\mathbf{B}^{\mathbf{0}}(\eta \zeta / 2)$ is a ball centered at the origin with radius $\eta \zeta / 2 . \quad \tilde{F}^{2}$ shows the representation of $\hat{F}^{2}$ in the coherent state formalism (see equation (2.9)). Using approximation in the first order of $\zeta$, the resulting metric will be as follows,

$$
\begin{aligned}
\widetilde{d s^{2}}= & \frac{1}{\rho^{2}}\left(1-\frac{\zeta}{\rho^{2}}+\mathcal{O}\left(\frac{\zeta}{\rho^{2}}\right)^{2}\right) d \vec{a}^{2} \\
& +\frac{1}{\rho^{2}}\left(1-\frac{2 \zeta}{\rho^{2}}+\mathcal{O}\left(\frac{\zeta}{\rho^{2}}\right)^{2}\right) d \rho^{2}
\end{aligned}
$$

In the following section we will discussed the above metric as a good candidate for the instanton metric which can be matched with the Supergravity.

The contribution of $H_{0}$ in (3.17) is in the second order of $\zeta$.

"Note that the cut-off $\eta$ will not appear in the first order of $\zeta$. 


\section{The AdS/CFT Correspondence}

In the context of String Theory, the non-commutativity can be traced in a background which contains anti-symmetric $B$ field. Here, we consider an Anti-Selfdual $B$ field extended in four directions and the following solution will be derived from the Supergravity equations in Euclidean Space-Time, [20, 7]

$$
\begin{aligned}
& d s^{2}=\frac{\alpha^{\prime} R^{2}}{\sqrt{\hat{g}}}\left[(f(u))^{-1 / 2}\left(d x_{0}^{2}+\cdots+d x_{3}^{2}\right)+(f(u))^{1 / 2}\left(d u^{2}+u^{2} d \Omega_{5}^{2}\right)\right] \\
& B_{01}=B_{23}=-\frac{i \alpha^{\prime} a^{2} R^{2}}{\hat{g}}(f(u))^{-1}
\end{aligned}
$$

where $R$ is the radius of $\mathrm{AdS}_{5}, \hat{g}$ is the normalized String coupling and

$$
f(u)=a^{4}+\frac{1}{u^{4}}
$$

The non-commutativity can be introduced as the parameter $a^{2}$ which is the inverse of $B$ in the limit $u \rightarrow \infty$. So we take $a^{2}=\lambda \zeta$, where $\lambda$ is a constant to control the $B$ field and $\zeta$ independently. Firstly, let us change the coordinate $u$ to $w=1 / u$ which the latter has the length dimension,

$$
d s^{2}=\frac{\alpha^{\prime} R^{2}}{\sqrt{\hat{g}}}\left[\left(1+a^{4} w^{4}\right)^{-1 / 2} \frac{d \vec{x}^{2}}{w^{2}}+\left(1+a^{4} w^{4}\right)^{1 / 2} \frac{d w^{2}}{w^{2}}\right]
$$

On the other hand, to compare our Informations Metrics (3.19) and (3.21) in the previous section with the metric (4.4), we consider the following change of variables,

$$
w=\beta \rho\left(1+\beta_{1} \frac{\zeta}{\rho^{2}}+\beta_{2}\left(\frac{\zeta}{\rho^{2}}\right)^{2}+\cdots\right)
$$

where $\beta$ and $\beta_{i}$ 's are arbitrary constants. Substituting (4.5) in the metric (4.4) will give the following result:

$$
\begin{aligned}
d s^{2}=\frac{\alpha^{\prime} R^{2}}{\sqrt{\hat{g}}} & {\left[\frac{1}{\beta^{2} \rho^{2}}\left(1-\frac{2 \beta_{1} \zeta}{\rho^{2}}-\frac{\left(-6 \beta_{1}^{2}+4 \beta_{2}+\lambda^{2}\right) \zeta^{2}}{2 \rho^{4}}+\mathcal{O}\left(\frac{\zeta}{\rho^{2}}\right)^{3}\right) d \vec{x}^{2}\right.} \\
+ & \left.\frac{1}{\rho^{2}}\left(1-\frac{4 \beta_{1} \zeta}{\rho^{2}}+\frac{\left(16 \beta_{1}^{2}-16 \beta_{2}+\lambda^{2}\right) \zeta^{2}}{2 \rho^{4}}+\mathcal{O}\left(\frac{\zeta}{\rho^{2}}\right)^{3}\right) d \rho^{2}\right]
\end{aligned}
$$

Note that $\lambda$ does not appear in the first order of $\zeta$. Indeed, up to the first order term in $\zeta / \rho^{2}$, choosing the following parameter will map both metrics (4.1) and (3.3) to the metric (3.21) resulting from Information Metric on the Instanton Moduli Space:

$$
a^{\mu}=\frac{x^{\mu}}{\beta} \quad \beta_{1}=\frac{1}{2}
$$

However, note that in this way, it is impossible to map the metric (3.19) to the metric in (4.4). So, it seems that only the modified metric in (3.21) is compatible with Supergravity at least, up to first order in $\zeta$. 


\section{$5 \quad$ Summary and Conclusion}

In the commutative background a map between YM-Instantons and gravity D-Instantons Moduli can be obtained directly by the Information Metric on the YM-Instanton Moduli [4]. We investigated a possible extension of the Information Metric to the noncommutative instantons. Firstly, we defined the Metric by simply replacing the instanton density by its operator version in the NC theory. This Metric can be calculated perturbatively in the non-commutativity parameter, $\zeta$. In the first order of $\zeta$, the naive definition of the metric is not in agreement with the Supergravity result.

We modified the definition by putting a cut-off around the location of instanton. This cut-off is a ball around the instanton with radius in order of $\zeta$. Now the modified Information Metric is compatible with the Maldacena-Russo type solutions in the supergravity [6, 7], at least in the first order of $\zeta$.

The issue of higher order matching can shed more light on this idea that the (NC) $Y M$ Instanton moduli space is isomorphic to the bulk space in the context of Gauge/Gravity correspondence. Note that this correspondence is different from the ordinary AdS/CFT since a) we are not using the conventional $L^{2}$ metric in the field configuration space, even in the commutative case, and b) this correspondence works for small rank and small instanton number.

The next important issue can be the multi-instanton moduli space. It can be restricted to the simple situation of dilute gas of instantons e. g. two separated instantons or very close two instantons for the latter case the commutative moduli is singular but the noncommutative moduli space can be considered as a resolution of the singularity and the Information Metric may have an interesting interpretation.

\section{Acknowledgment}

The author is very grateful to Gautum Mandal and Spenta Wadia for very fruitful discussions and reading the manuscript. Thanks also to Anindya k. Biswas, Avinash Dhar, Rajesh Gopakumar and Sandip Trivedi for useful comments and discussions. This work was partially supported by a travel grant from the Institute for Studies in Theoretical Physics and Mathematics, Tehran, Iran.

\section{References}

[1] O. Aharony, S. Gubser, J. Maldacena, H. Ooguri, Y Oz, "Large $N$ Field Theories, String Theory and Gravity", Phys. Rept. 323 (2000) 183, hep-th/9905111.

[2] N. Dorey, V.V. Khoze, M.P. Mattis, S. Vandoren, "Yang-Mills Instantons in the Large $N$ limit and the AdS/CFT Correspondence", Phys.Lett. B442 (1998) 145, hepth/9808157;

N. Dorey, T.J. Hollowood, V.V. Khoze, M.P. Mattis, S. Vandoren, "Multi-Instantons and Maldacena's Conjecture", JHEP 9906 (1999) 023, hep-th/9810243; "Multi- 
Instanton Calculus and the AdS/CFT Correspondence in $N=4$ Superconformal Field Theory", Nucl. Phys. B552 (1999) 88, hep-th/9901128.

[3] N.J. Hitchin, "The Geometry and Topology of Moduli Spaces", in Global Geometry and Mathematical Physics, Lecture Notes in Mathematics 1451, Springer, Heidelberg (1988) 1.

[4] M. Blau, K.S. Narain, G. Thompson, "Instantons, the Information Metric, and the AdS/CFT Correspondence", hep-th/0108122.

[5] D. Grossier, M.K. Murray, "Instantons and the In formation Metric", Ann. Global Anal. Geom. 15 (1997) 519, dg-ga/9611008.

[6] J. Maldacena, J.G. Russo, "Large N Limit of Noncommutative Gauge Theories", Class. Quantum. Grav. 17 (2000) 1189.

[7] S.R. Das, S.K. Rama and S.P. Trivedi, "Supergravity with Self-dual B fields and Instantons in Noncommutative Gauge Theory", JHEP 0003 (2000) 004, hepth/9911137.

[8] M.F. Atiyah, N.J. Hitchin, V.G. Drinfeld, Yu.I. Manin, "Construction of Instantons", Phys. Lett. A65 (1978) 185.

[9] N. Nekrasov and A. Schwarz, "Instantons on noncommutative $\mathbf{R}^{\mathbf{4}}$, and (2,0) superconformal six dimensional theory", hep-th/9802068.

[10] K. Furuuchi, "Instantons on Noncommutative $R^{4}$ and Projection Operators", Prog. Theor. Phys. 103 (2000) 1043 hep-th/9912047.

[11] K. Lee, D. Tong and S. Yi, "The moduli space of two U(1) instantons on noncommutative $R^{4}$ and $R^{3} \times S^{1}$ ", Phys.Rev. D63 (2001) 065017, hep-th/0008092.

[12] K.Y. Kim, B.H. Lee and H. S. Yang, "Noncommutative Instantons on $R_{N C}^{2} \times R_{C}^{2}$ ", Phys.Lett. B523 (2001) 357, hep-th/0109121.

[13] D.H. Correa, G. Lozano, E.F. Moreno and F.A. Schaposnik, "Comments on the U(2) Noncommutative Instanton", Phys.Lett. B515 (2001) 206, hep-th/0105085.

[14] K.Y. Kim, B.H. Lee and H. S. Yang, "Comments on Instantons on Noncommutative $\mathbf{R}^{4}$ ", hep-th/0003093.

[15] C.S. Chu, V.V. Khoze and G. Travaglini, "Notes on Noncommutative Instantons", Nucl. Phys. B621 (2002) 101, hep-th/0108007.

[16] M. Hamanaka, "ADHM/Nahm construction of localized solitons in noncommutative gauge theories", hep-th/0109070.

[17] O. Lechtenfeld and A.D. Popov, "Noncommutative 't Hooft Instantons", hepth/0109209. 
[18] T. Ishikawa, S. Kuroki and A. Sako, "Instanton Number Calculus on Noncommutative $R^{4}$, hep-th/0201196.

[19] N. Seiberg and E. Witten, JHEP 9909 (1999) 032, hep-th/9908142.

[20] J.M. Maldacena and J.G. Russo, "Large N Limit of Noncommutative Gauge Theories", JHEP 9909 (1999) 025, hep-th/9908134. 